\title{
Accuracy of non-invasive body temperature measurement methods in critically ill patients: a prospective, bicentric, observational study
}

Salvatore L Cutuli, Eduardo A Osawa, Christopher T Eyeington, Helena Proimos, Emmanuel Canet, Helen Young, Leah Peck, Glenn M Eastwood, Neil J Glassford, Michael Bailey and Rinaldo Bellomo

Body temperature (BT) is strictly regulated ${ }^{1-3}$ and is a key vital sign. In critically ill patients, abnormal BT is associated with adverse clinical outcomes. ${ }^{4,5}$ Moreover, BT abnormalities are used to identify specific phenotypes of immune reaction to infection ${ }^{6}$ and to evaluate response to interventions (eg, antibiotic or anti-inflammatory therapies). ${ }^{7}$ Accordingly, prompt recognition and monitoring of BT perturbations is fundamental in the management of traumatic brain injury ${ }^{8}$ and cardiac arrest ${ }^{9}$ and also as an early warning sign of critically ill patients in general.

The pulmonary artery catheter is considered the gold standard for the measurement of core temperature, ${ }^{10}$ but its use is limited and declining. ${ }^{11,12}$ Compared with pulmonary artery catheter, temperature-sensing intra-urinary bladder catheters, oesophageal and nasopharyngeal probes may represent less invasive methods of core BT measurement. However, their use is limited in critical care practice due to urine output dependence and easy displacement. Thus, non-invasive BT measurement methods (axillary thermometer, tympanic infrared and temporal scanner) have become common. 2,13-15 But their accuracy in estimating core BT in ICU patients is uncertain. ${ }^{16,17}$ Moreover, a recent survey ${ }^{18}$ highlighted a lack of agreement on the preferred BT measurement methods between doctors and nurses and lack of protocol governance among Australian and New Zealand ICUs.

Accordingly, we sought to investigate the accuracy of the axillary chemical dot, tympanic infrared and temporal scanner methods in estimating core temperature in critically ill patients admitted to the ICU. The primary hypothesis was that all three noninvasive BT measurement methods would show low accuracy in estimating core BT compared with invasive methods and that these accuracies would differ from each other. The secondary hypothesis was that accuracy of each non-invasive BT measurement method would be influenced by clinical variables.

\begin{abstract}
Objective: The accuracy of different non-invasive body temperature measurement methods in intensive care unit (ICU) patients is uncertain. We aimed to study the accuracy of three commonly used methods.

Design: Prospective observational study.

Setting: ICUs of two tertiary Australian hospitals.

Participants: Critically ill patients admitted to the ICU.

Interventions: Invasive (intravascular and intra-urinary bladder catheter) and non-invasive (axillary chemical dot, tympanic infrared, and temporal scanner) body temperature measurements were taken at study inclusion and every 4 hours for the following 72 hours.
\end{abstract}

Main outcome measures: Accuracy of non-invasive body temperature measurement methods was assessed by the BlandAltman approach, accounting for repeated measurements and significant explanatory variables that were identified by regression analysis. Clinical adequacy was set at limits of agreement (LOA) of $1^{\circ} \mathrm{C}$ compared with core temperature.

Results: We studied 50 consecutive critically ill patients who were mainly admitted to the ICU after cardiac surgery. From over 375 observations, invasive core temperature (mostly pulmonary artery catheter) ranged from $33.9^{\circ} \mathrm{C}$ to $39^{\circ} \mathrm{C}$. On average, the LoA between invasive and non-invasive measurements methods were about $3^{\circ} \mathrm{C}$. The temporal scanner showed the worst performance in estimating core temperature (bias, $0.66^{\circ} \mathrm{C}$; LoA, $-1.23^{\circ} \mathrm{C},+2.55^{\circ} \mathrm{C}$ ), followed by tympanic infrared (bias, $0.44^{\circ} \mathrm{C}$; LoA, $-1.73^{\circ} \mathrm{C},+2.61^{\circ} \mathrm{C}$ ) and axillary chemical dot methods (bias, $0.32^{\circ} \mathrm{C}$; LoA, $-1.64^{\circ} \mathrm{C},+2.28^{\circ} \mathrm{C}$ ). No methods achieved clinical adequacy even accounting for significant explanatory variables.

Conclusions: The axillary chemical dot, tympanic infrared and temporal scanner methods are inaccurate measures of core temperature in ICU patients. These non-invasive methods appeared unreliable for use in ICU patients.

Crit Care Resusc 2021; 23 (3): 346-53 


\section{ORIGINAL ARTICLE}

\section{Methods}

The Ethics of Human Research Committee of the Austin Hospital approved the study and waived the need for informed consent (LNR/17/Austin/315) due to its implied observational nature.

\section{Study design and population}

We performed a prospective observational study in critically ill patients admitted to the ICU of two tertiary metropolitan hospitals (Austin Hospital and Warringal Hospital, Melbourne, VIC). We included patients who had a temperature-sensing intravascular (mostly pulmonary artery catheter) or intra-urinary bladder catheter, which was placed before the inclusion in the study by the treating physician. We excluded patients aged less than 18 years, patients with skin infection, and pregnant women

\section{BT measurement methods}

Characteristics (manufacturer and other commercial details) of the BT measurement methods used in the study are reported in the Online Appendix, eTable1. All BT measurement methods were calibrated according to hospital protocols and manufacturer's specifications. We classified BT measurement methods as:

- Invasive: temperature-sensing intravascular or intraurinary bladder catheters. Intravascular catheters included temperature-sensing vascular probe placed into the pulmonary artery or the femoral artery (pulse contour cardiac output, PiCCO). The correct position of the pulmonary artery catheter was as described. ${ }^{19}$

- Non-invasive: axillary chemical dot, temporal scanner and tympanic infrared methods.

Concurrent invasive and non-invasive BT measurements were taken by on-duty nurses at the inclusion in the study and every 4 hours for the following 72 hours, as per hospital protocol. Intra-urinary bladder temperature measurements were recorded only when an intravascular catheter was not in place. Due to analogue accuracy in estimating core temperature, ${ }^{20}$ intra-urinary bladder and intravascular measurements were merged and reported as "invasive BT measurement methods".

\section{Data collection}

Patients' demographic data (age, sex, height and weight), cardiovascular comorbidities and main pathology at ICU admission were recorded. Concurrent haemodynamic parameters (blood pressure and heart rate), medications (sedatives, opioids, vasopressors and inotropes), fluid balance, artificial organ supports (mechanical ventilation, renal replacement therapy and extracorporeal membrane oxygenation), and warming or cooling interventions were recorded at each BT measurement time point.

\section{Outcomes}

The primary outcome of the study was the assessment of accuracy of non-invasive BT measurement methods in estimating core temperature and the relationship between axillary chemical dot, tympanic infrared and temporal scanner methods by the evaluation of:

- Bias: mean difference between temperature measured by invasive methods and either axillary chemical dot, tympanic infrared or temporal scanner. Bias was used to express accuracy, as fixed offset between BT measurements.

- Limits of agreement (LoA): 95\% confidence interval (CI) of the differences between body temperature measured by invasive methods and either axillary chemical dot, tympanic infrared or temporal scanner.

Specifically, clinical accuracy was defined as bias between each method and the others within $\pm 0.2^{\circ} \mathrm{C} .{ }^{16}$ Clinical adequacy was set at LoA of $1^{\circ} \mathrm{C}$ compared with core temperature.

A secondary outcome of the study was the evaluation of clinical conditions or interventions, which predicted bias and LoA between invasive and non-invasive BT measurements.

\section{Statistical analysis}

Continuous data are presented as mean (standard error; SE) or median (interquartile range; IQR), categorical data are summarised as number (percentage). After assessing the distribution of the data with the kernel density plot and the Kolmogorov-Smirnov test, the relationship between invasive and non-invasive methods was investigated with the Bland-Altman approach, by plotting differences versus means of paired core temperature (gold standard) and noninvasive (index methods) measurements. 21-25

To account for repeated measurements of BT in the same individual and adjust for significant explanatory clinical variables, mixed linear modelling was performed and patients' demographic characteristics, comorbidities, medications and extracorporeal organ supports were included in a multivariable model. Then, bias and LoA were adjusted for significant predictors of BT temperature. Finally, a multivariate analysis accounting for repeated measures was performed with the aim to explore the relationship between variables which were independently associated with BT and bias of non-invasive BT measurement methods in estimating core temperature. We analysed data using Stata/SE 13 (StataCorp, College Station, TX, USA) and SAS version 9.4 (SAS Institute Cary, NC, USA) while graphs were plotted using MS Excel 2017 (Microsoft, Seattle, WA). To increase the robustness of this study, a reduced two-sided $P$ value of 0.01 was used to indicate statistical significance. 


\begin{tabular}{|lc|}
\hline Table 1. Patients' demographic characteristics at inclusion \\
Variable & Patients \\
\hline Total number of patients & 50 \\
\hline Age (years), median (IQR) & $68(59-74)$ \\
\hline Sex, male & $35(70 \%)$ \\
\hline Weight (kg), median (IQR) & $76.4(70-95)$ \\
\hline Height (cm), median (IQR) & $170(163-173)$ \\
\hline APACHE III score, median (IQR) & $40(33-49)$ \\
\hline Comorbidities & \\
\hline Coronary artery disease & $31(62 \%)$ \\
Hypertension & $27(54 \%)$ \\
\hline Dyslipidaemia & $22(44 \%)$ \\
Smoke & $28(56 \%)$ \\
\hline Sedatives* & $50(100 \%)$ \\
\hline Opioids & $7(14 \%)$ \\
\hline Neuromuscular blockers & $2(4 \%)$ \\
\hline Mechanical ventilation & $47(94 \%)$ \\
\hline Haemodynamic support & $2(4 \%)$ \\
\hline Vasopressors/inotropes & $13(26 \%)$ \\
\hline Extracorporeal membrane oxygenation & $1(2 \%)$ \\
\hline Renal replacement therapy & $3(6 \%)$ \\
\hline Cardiac surgery & \\
\hline Respiratory failure & \\
\hline Liver failure & \\
\hline
\end{tabular}

Table 2. Accuracy of non-invasive measurement methods accounting for repeated measures

\begin{tabular}{|c|c|c|c|c|}
\hline Methods & $n$ & $\begin{array}{c}\text { Mean difference, }{ }^{\circ} \mathrm{C} \\
(95 \% \mathrm{Cl})\end{array}$ & $\begin{array}{l}\text { 95\% Limits of } \\
\text { agreement } \\
\text { (lower, } \\
\text { upper) }\end{array}$ & $P$ \\
\hline Invasive - axillary chemical dot & 279 & $0.32(0.21-0.43)$ & $-1.64,2.28$ & $<0.001$ \\
\hline Invasive - temporal scanner & 361 & $0.66(0.56-0.76)$ & $-1.23,2.55$ & $<0.001$ \\
\hline Invasive - tympanic infrared & 285 & $0.44(0.32-0.56)$ & $-1.73,2.61$ & $<0.001$ \\
\hline $\begin{array}{l}\text { Axillary chemical dot - temporal } \\
\text { scanner }\end{array}$ & 275 & $0.34(0.24-0.44)$ & $-1.34,2.02$ & $<0.001$ \\
\hline $\begin{array}{l}\text { Axillary chemical dot - tympanic } \\
\text { infrared }\end{array}$ & 279 & $0.12(0.01-0.24)$ & $-1.86,2.11$ & 0.041 \\
\hline Tympanic - temporal & 290 & $0.22(0.11-0.33)$ & $-1.70,2.13$ & $<0.001$ \\
\hline
\end{tabular}

\section{Results}

\section{Patients}

A convenience sample of 50 consecutive critically ill patients ( $n=35,70 \%$ male) was included in the study. Demographic and clinical characteristics of all patients are shown in Table 1. Median age was 68 years (IQR, 59-74 years) and median APACHE III score was 40 (IQR, 33-49). The most frequent reason for ICU admission was cardiac surgery ( $n=44,88 \%)$. All patients were sedated $(N=50$, $100 \%)$, most were mechanically ventilated ( $n=47$, $94 \%$ ) and 13 patients (26\%) were receiving vasopressor/inotropic support at study inclusion.

\section{Primary outcome}

Over 375 observations in 50 patients, core temperature measured by invasive BT measurement method ranged between $33.9^{\circ} \mathrm{C}$ and $39^{\circ} \mathrm{C}$ (Online Appendix, eTable 2). Non-invasive BT measurement methods systematically underestimated core temperature (Table 2). On average, the LoA between invasive and non-invasive BT measurements methods was about $3^{\circ} \mathrm{C}$, with the temporal scanner methods showing the worst performance in estimating core temperature (bias, $0.66^{\circ} \mathrm{C}$; LoA, $\left.-1.23^{\circ} \mathrm{C},+2.55^{\circ} \mathrm{C}\right)$, followed by tympanic infrared (bias, $0.44^{\circ} \mathrm{C}$; LoA, $-1.73^{\circ} \mathrm{C},+2.61^{\circ} \mathrm{C}$ ) and the axillary chemical dot method (bias, $0.32^{\circ} \mathrm{C}$; LoA, $\left.-1.64^{\circ} \mathrm{C},+2.28^{\circ} \mathrm{C}\right)$. The axillary chemical dot and tympanic infrared had the lowest degree of bias and the largest LoA, which approximated $4^{\circ} \mathrm{C}$. However, none of the non-invasive BT measurement methods showed clinically acceptable accuracy.

\section{Secondary outcome}

The regression model accounting for repeated measures of BT is presented in the Online Appendix, eTable 3. An independent and negative association was found between BT and propofol infusion, mechanical ventilation, neuromuscular blocker agents, renal replacement therapy, aortic surgery and active warming. After adjusting for significant covariates 
Figure 1. Bland-Altman plots of temperature measurements, accounting for repeated measures and adjusted for significant covariates: (A) invasive and axillary chemical dot method, (B) invasive and tympanic infrared method, and (C) invasive and temporal scanner method

A

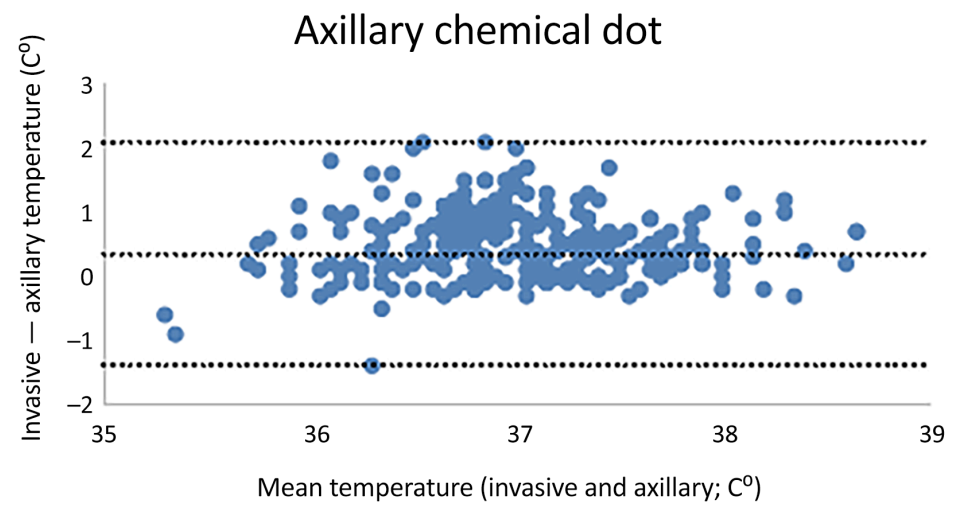

B

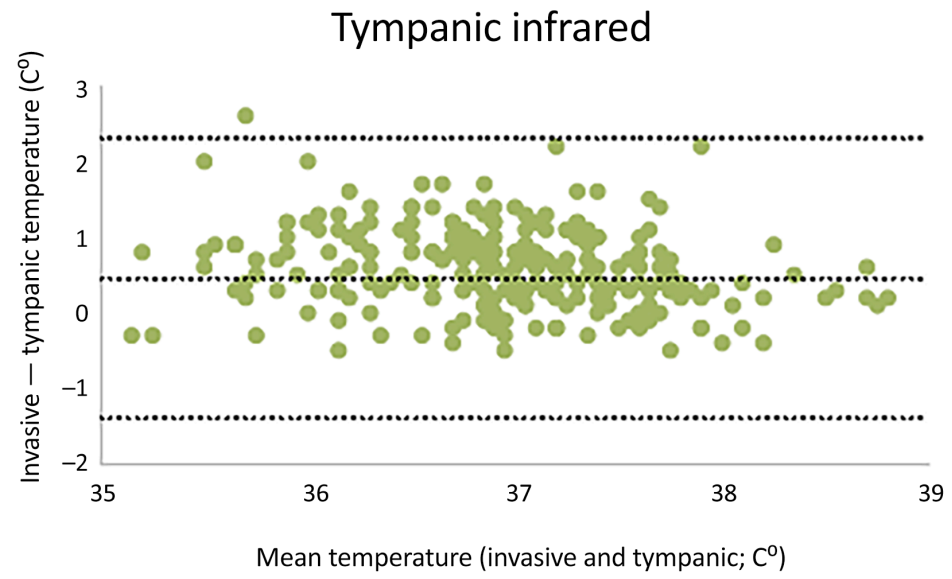

C

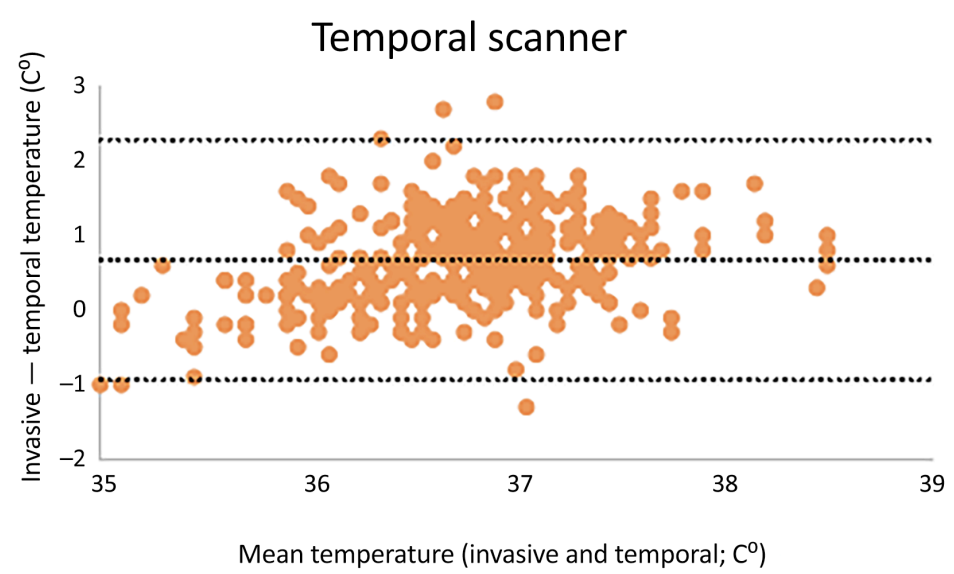

Each coloured dot corresponds to mean (x-axis) and difference (y-axis) of paired invasive (gold standard) and non-invasive (index methods) measurements. Among horizontal dotted lines, the inner represents the mean difference (bias) and the outers represent the $95 \% \mathrm{Cl}$ of differences (limit of agreement) of paired invasive (gold standard) and non-invasive (index methods) measurements.
(Online Appendix, eTable 4), bias and LoA between invasive and non-invasive BT measurement methods were reduced (Online Appendix, eTable 5). However, none of the non-invasive BT measurement methods showed clinically acceptable accuracy in estimating core temperature. The corresponding Bland-Altman plots are represented in Figure 1, A-C. Moreover, we reported the deviation of each non-invasive BT measurements from invasive temperature (Online Appendix, efigure 1, A-C).

The regression model exploring the relationship between explanatory variables of BT and bias between invasive and non-invasive BT measurement methods is presented in Table 3. None of the clinical variables included in the analysis showed significant association with bias either between core temperature and tympanic infrared or temporal scanner.

Axillary chemical dot failed to measure BT in 16 observations and was reported by nurses as non-readable. Tympanic infrared failed once in measuring BT (Online Appendix, eTable 2).

\section{Discussion}

\section{Key findings}

We assessed the accuracy of the axillary chemical dot, tympanic infrared and temporal scanner methods to estimate core temperature in ICU patients. Each of the non-invasive BT measurement methods showed wide LOA and inadequate clinical accuracy in estimating core temperature among an average of more than 300 paired observations. Moreover, each non-invasive BT measurement 
Table 3. Predictors of bias between invasive and non-invasive body temperature measurement methods accounting for repeated measures

\begin{tabular}{|c|c|c|c|c|c|c|}
\hline \multirow[b]{2}{*}{ Model } & \multicolumn{3}{|c|}{ Univariate analysis* } & \multicolumn{3}{|c|}{ Multivariable analysis* } \\
\hline & Estimate & $\begin{array}{c}95 \% \mathrm{Cl} \\
\text { (lower, upper) }\end{array}$ & $P$ & Estimate & $\begin{array}{c}95 \% \mathrm{Cl} \\
\text { (lower, upper) }\end{array}$ & $P$ \\
\hline \multicolumn{7}{|l|}{$\begin{array}{l}\text { Fixed effect: invasive } v \text { axillary } \\
\text { chemical dot }\end{array}$} \\
\hline \multicolumn{7}{|l|}{ Covariates } \\
\hline Propofol & -0.001 & $-0.003,0.001$ & 0.15 & -0.0001 & $-0.001,0.001$ & 0.98 \\
\hline Active warming & -0.16 & $-0.55,0.23$ & 0.42 & -0.01 & $-0.37,0.36$ & 0.96 \\
\hline Mechanical ventilation & -0.24 & $-0.42,-0.07$ & 0.007 & -0.22 & $-0.39,-0.04$ & 0.02 \\
\hline Neuromuscular blocking agents & -0.004 & $-0.33,0.32$ & 0.98 & 0.15 & $-0.15,0.46$ & 0.33 \\
\hline Renal replacement therapy & -0.05 & $-0.40,0.30$ & 0.79 & -0.03 & $-0.35,0.30$ & 0.87 \\
\hline Aortic surgery & -0.34 & $-1.46,0.77$ & 0.55 & -0.45 & $-1.54,0.63$ & 0.41 \\
\hline Enrolment time point & 0.014 & $-0.002,0.03$ & 0.06 & 0.01 & $-0.002,0.02$ & 0.09 \\
\hline \multicolumn{7}{|l|}{$\begin{array}{l}\text { Fixed effect: invasive } v \text { tympanic } \\
\text { infrared }\end{array}$} \\
\hline \multicolumn{7}{|l|}{ Covariates } \\
\hline Propofol & 0.0003 & $-0.002,0.002$ & 0.55 & 0.0003 & $-0.001,0.001$ & 0.53 \\
\hline Active warming & -0.12 & $-0.44,0.20$ & 0.48 & -0.05 & $-0.35,0.25$ & 0.72 \\
\hline Mechanical ventilation & -0.04 & $-0.20,0.13$ & 0.65 & -0.05 & $-0.21,0.12$ & 0.60 \\
\hline Neuromuscular blocking agents & 0.17 & $-0.15,0.48$ & 0.30 & 0.21 & $-0.09,0.51$ & 0.17 \\
\hline Renal replacement therapy & -0.04 & $-0.39,0.30$ & 0.81 & -0.05 & $-0.37,0.27$ & 0.75 \\
\hline Aortic surgery & -0.34 & $-1.14,0.45$ & 0.40 & -0.36 & $-1.18,0.46$ & 0.39 \\
\hline Enrolment time point & -0.002 & $-0.02,0.12$ & 0.74 & 0.001 & $-0.01,0.01$ & 0.93 \\
\hline \multicolumn{7}{|l|}{$\begin{array}{l}\text { Fixed effect: invasive } v \text { temporal } \\
\text { scanner }\end{array}$} \\
\hline \multicolumn{7}{|l|}{ Covariates } \\
\hline Propofol & -0.002 & $-0.003,-0.001$ & 0.01 & -0.001 & $-0.002,0.001$ & 0.20 \\
\hline Active warming & -0.40 & $-0.71,-0.08$ & 0.01 & -0.31 & $-0.61,0.01$ & 0.045 \\
\hline Mechanical ventilation & -0.08 & $-0.23,0.07$ & 0.28 & -0.01 & $-0.17,0.15$ & 0.88 \\
\hline Neuromuscular blocking agents & 0.08 & $-0.33,0.49$ & 0.71 & 0.07 & $-0.29,0.42$ & 0.71 \\
\hline Renal replacement therapy & -0.02 & $-0.44,0.40$ & 0.93 & 0.01 & $-0.35,0.38$ & 0.94 \\
\hline Aortic surgery & -0.88 & $-1.37,-0.38$ & 0.001 & -0.72 & $-1.32,-0.11$ & 0.02 \\
\hline Enrolment time point & 0.014 & $-0.002,0.03$ & 0.08 & 0.004 & $-0.01,0.02$ & 0.58 \\
\hline
\end{tabular}

method showed low agreement with other non-invasive methods. Finally, adjusting for explanatory variables of BT such as propofol infusion, mechanical ventilation, neuromuscular blocker agents, renal replacement therapy, aortic surgery and active warming only improved their accuracy to a limited degree.

\section{Relationship to previous studies}

Previous international guidelines published in $2008^{26}$ suggested avoidance of axillary methods, temporal scanner and chemical dot thermometers in ICU patients. However, such recommendations were only supported by observational studies $27-31$ with controversial findings and methodological problems. Specifically, there was lack of control for possible confounders such as device calibration, investigator skill assessment, method used, failure to use the Bland-Altman approach in estimating bias and LoA, and lack of adjustment for multiple within-subject measurement. ${ }^{32}$ Thus, these recommendations do not appear to have been widely adopted. 


\section{ORIGINAL ARTICLE}

These shortcomings were further highlighted in an initial systematic review. ${ }^{16}$ In a further systematic review of 75 studies in adult and paediatric patients from different clinical settings (only $45 \% \mathrm{ICU}$ ), investigators concluded that peripheral thermometers do not have clinically acceptable accuracy, although high quality data were limited and study heterogeneity was significant. In a more recent systematic review of 13 studies in adult ICU patients, Cutuli and colleagues $^{32}$ found that the majority of studies did not control for clinical confounders ${ }^{33}$ or used suboptimal statistical methods. ${ }^{21-24}$ Because of such limitations and the wide types of non-invasive thermometry used to assess BT, both systematic reviews ${ }^{16,32}$ could not synthesise the data available and/or obtain conclusive results. Given the above problems, we aimed to overcome such methodological issues.

Our findings are similar to and extend those of Moran et al, ${ }^{20}$ who investigated the accuracy of tympanic infrared and axillary glass mercury thermometers in estimating core temperature in a cohort of critically ill patients. In their study, the accuracy of the axillary glass mercury method was superior to the tympanic infrared method. However, the authors did not specify whether non-invasive BT measurement methods had been calibrated before the commencement of the study and set tympanic infrared in "core mode". In our study, the tympanic infrared devices were unadjusted to assess core temperature according to the manufacturer algorithm ${ }^{4}$ (ear mode $+1.04^{\circ} \mathrm{C}$ ). However, such conversion would have only influenced the direction of the bias, while the magnitude of inaccuracy would have remained unchanged.

In contrast with our observations, Fulbrook and colleagues $^{34}$ reported that axillary chemical dots slightly underestimated core temperature compared with the tympanic infrared method. However, the authors only included a small number of patients and observations. More recently, Farnell et al ${ }^{25}$ reported axillary chemical dot failures in seven of 160 BT measurements in 25 critically ill patients, which confirms the rate of "non-readable" strips seen in our study. In addition, axillary chemical dots appeared to show lower accuracy than the tympanic infrared method in estimating core temperature. However, as the authors did not account for repeated within-subject measurements, the robustness of such findings is unclear. Finally, Myny et $\mathrm{al}^{35}$ argued in favour of a better accuracy for the temporal scanner in estimating core temperature in 57 critically ill patients. Yet, the number of observations was small and the participation of only one operator increased observer bias and limited external validity.

\section{Clinical implications}

The low accuracy of axillary chemical dot, tympanic infrared and temporal scanner methods in estimating core temperature implies that these methods are clinically inadequate for ICU patients. Such implication is of importance for research in ICU patients with specific conditions (eg, suspected infection), for whom BT may represent an inclusion criterion. Moreover, the wide LoA imply that "average" adjustments upward of 0.5 or 1 degree are not helpful. In addition, the impact on bias and LoA of clinical explanatory variables implies that any link between these techniques and core temperature will markedly vary according to clinical circumstances. Finally, the high rate of failure for the chemical dot approach is of concern and implies limited applicability. In their aggregate, our findings imply that current non-invasive temperature measurement techniques are inappropriate and potentially misleading in many ICU patients.

\section{Strengths and limitations}

Our study has several strengths. First, we investigated the accuracy of the most widely used non-invasive BT measurement methods in ICU. Second, we used the BlandAltman approach accounting for repeated within-subject measures, which allowed us to overcome methodological issues raised by previous systematic reviews. ${ }^{16,32}$ Third, we performed regression analysis to identify predictors of BT, which allowed us to explore the impact of clinical variables on temperature estimation. Nonetheless, our study carries some limitations. It was not blinded and onduty bedside nurses may have been biased in their data collection. However, nurses were asked to measure BT as they do in daily clinical practice and we estimate that more than 100 nurses were involved in the study measurements, which gives our findings a robust level of external validity and makes systematic bias unlikely. We tested only few non-invasive BT measurement methods and our results do not apply to other types of thermometry such as the newest zero heat flux. ${ }^{36,37}$ However, such thermometers are of limited daily use because of their cost. We found wider LoA compared with previous articles in this field, ${ }^{32}$ supporting the limited use of non-invasive BT measurement methods in the ICU. However, this finding may be explained by the design of our study, which controlled for clinical confounders and adopted adequate statistical method to account for significant explanatory variables and multiple within-subject measurements. Finally, we merged different invasive BT methods into a single variable; however, previous evidence $^{20}$ investigated the accuracy of temperaturesensing urinary catheters in estimating core temperature and confirmed their negligible bias and LoA.

\section{Conclusions}

Non-invasive body temperature measurement techniques are inaccurate, show bias and have wide LoA in relation 


\section{ORIGINAL ARTICLE}

to core temperature in ICU patients. Given the diagnostic and therapeutic importance of this vital sign in critically ill patients, the use of axillary chemical dot, tympanic infrared and temporal scanner methods appears suboptimal in the ICU.

Acknowledgements: We thank the ICU nurses who work at the Austin Hospital and Warringal Private Hospital. This research did not receive any specific grant from funding agencies in the public, commercial, or not-for-profit sectors.

\section{Competing interests}

No relevant disclosures.

\section{Author details}

Salvatore L Cutuli1,2
Eduardo A Osawa $^{1}$
Christopher T Eyeington $^{1}$
Helena Proimos $^{1}$
Emmanuel Canet $^{1}$
Helen Young
Leah Peck
Glenn M Eastwood
Neil J Glassford
Michael Bailey
B,5
Rinaldo Bellomo

1 Department of Intensive Care, Austin Hospital, Melbourne, VIC, Australia.

2 Dipartimento di Scienze dell'Emergenza, Anestesiologiche e della Rianimazione, Fondazione Policlinico Universitario A. Gemelli IRCCS, Rome, Italy.

3 Australian and New Zealand Intensive Care Research Centre, School of Public Health and Preventive Medicine, Monash University, Melbourne, VIC, Australia.

4 Department of Intensive Care, Royal Melbourne Hospital, Melbourne Health, Melbourne, VIC, Australia.

5 Centre for Integrated Critical Care, University of Melbourne, Melbourne, VIC, Australia.

Correspondence: salvatorelucio.cutuli@policlinicogemelli.it

doi: https://doi.org/10.51893/2021.3.0A12

\section{References}

1 Faulds M, Meekings T. Temperature management in critically ill patients. Anaesthesia Continuing Education in Anaesthesia Critical Care and Pain 2013; 13: 75-9.

2 Sessler D. Temperature monitoring and perioperative thermoregulation. Anesthesiology 2008; 109: 318-38.
3 Chacko B, Peter J. Temperature monitoring in the intensive care unit. Indian J Respir Care 2018; 7: 28-32.

4 Peres Bota D, Lopes Ferreira F, Mélot C, et al. Body temperature alterations in the critically ill. Intensive Care Med 2004; 30: 811-6.

5 Laupland K, Zahar J, Adrie C, et al. Determinants of temperature abnormalities and influence on outcome of critical illness. Crit Care Med 2012; 40: 145-51.

6 Bhavani S, Wolfe K, Hrusch C, et al. Temperature trajectory subphenotypes correlate with immune responses in patients with sepsis. Crit Care Med 2020; 48: 1645-53.

7 Sajadi $M$, Bonabi R, Sajadi $M$, et al. Akhawayn and the first fever curve. Clin Infect Dis 2012; 55: 976-80.

8 Carney N, Totten A, O'Reilly C, et al. Guidelines for the management of severe traumatic brain injury, fourth edition. Neurosurgery 2017; 80: 6-15.

9 Nolan J, Soar J, Cariou A, et al. European Resuscitation Council and European Society of Intensive Care Medicine guidelines for post-resuscitation care 2015: section 5 of the European Resuscitation Council guidelines for resuscitation 2015. Resuscitation 2015; 95: 202-22.

10 Eichna L, Berger A, Rader B, et al. Comparison of intracardiac and intravascular temperatures with rectal temperatures in man. J Clin Invest 1951; 30: 353-9.

11 Wiener $\mathrm{R}$, Welch $\mathrm{H}$. Trends in the use of the pulmonary artery catheter in the United States, 1993-2004. JAMA 2007; 298: 423-9.

12 Pandey A, Khera R, Kumar N, et al. Use of pulmonary artery catheterization in US patients with heart failure, 2001-2012. JAMA Int Med 2016; 176: 129-32.

13 Hammond N, Saxena M, Taylor C, et al. Temperature management of non-elective intensive care patients without neurological abnormalities: a point prevalence study of practice in Australia and New Zealand. Crit Care Resusc 2013; 15: 228-33.

14 Saxena M, Taylor C, Hammond N, et al. Temperature management in patients with neurological lesions: an Australian and New Zealand point prevalence study. Crit Care Resusc 2013; 15: 110-8.

15 Saxena M, Colman T, Hammond N, et al. A multicentre audit of temperature patterns after traumatic brain injury. Crit Care Resusc 2015; 11: 129-34.

16 Jefferies $S$, Weatherall $M$, Young $P$, et al. A systematic review of the accuracy of peripheral thermometry in estimating core temperatures among febrile critically ill patients. Crit Care Resusc 2011; 13: 194-9.

17 Niven D, Gaudet J, Laupland K, et al. Accuracy of peripheral thermometers for estimating temperature. A systematic review and meta-analysis. Ann Inter Med 2016; 2015: 768-77.

18 Cutuli SL, Osawa EA, Glassford NJ, et al. Body temperature measurement methods and targets in Australian and New Zealand intensive care units. Crit Care Resusc 2018; 20: 241-4.

19 Shellock F, Rubin S. Simplified and highly accurate core temperature measurements. Med Prog Technol 1982; 8: 187-8. 


\section{ORIGINAL ARTICLE}

20 Moran J, Peter J, Solomon P, et al. Tympanic temperature measurements: are they reliable in the critically ill? A clinical study of measures of agreement. Crit Care Med 2007; 35: 155-64.

21 Bland J, Altman A. Statistical methods for assessing agreement between two methods of clinical measurement. Lancet 1986; 1: 307-10.

22 Bland J, Altman A. Measuring agreement in method comparison studies. Stat Methods Med Res 1999; 8: 135-60.

23 Bland J, Altman A. Comparing methods of measurement: why plotting difference against standard method is misleading. Lancet 1995; 346: 1085-7.

24 Bland J, Altman A. Agreement between methods of measurement with multiple observations per individual. J Biopharm Stat 2007; 17: 571-82.

25 Farnell S, Maxwell L, Tan A, et al. Temperature measurement: comparison of non-invasive methods used in adult critical care. J Clin Nurs 2003; 14: 632-9.

26 O'Grady N, Barie P, Bartlett J, et al. Guidelines for evaluation of new fever in critically ill adult patients: 2008 update from the American College of Critical Care Medicine and the Infectious Diseases Society of America. Crit Care Med 2008; 36: 1330-49.

27 Erickson R, Kirklin S. Comparison of ear-based, bladder, oral, and axillary methods for core temperature measurement. Crit Care Med 1993; 21: 1528-34.

28 Erickson R, Meyer L. Accuracy of infrared ear thermometry and other temperature methods in adults. Am J Crit Care 1994; 3: 40-54.
29 Schmitz T, Bair N, Falk M, et al. A comparison of five methods of temperature measurement in febrile intensive care patients. Am J Crit Care 1995; 4: 286-92.

30 Milewski A, Ferguson KL, Terndrup TE. Comparison of pulmonary artery, rectal, and tympanic membrane temperatures in adult intensive care unit patients. Clin Pediatr 1991; 34: 13-6.

31 Nierman D. Core temperature measurement in the intensive care unit. Crit Care Med 1991; 19: 818-23.

32 Cutuli SL, See EJ, Osawa EA, et al. Accuracy of non-invasive body temperature measurement methods in adult patients admitted to the intensive care unit: a systematic review and meta-analysis. Crit Care Resusc 2021; 23: 6-13.

33 Bridges E, Thomas K. Noninvasive measurement of body temperature in critically ill patients. Crit Care Nurse 2009; 29: 94-7.

34 Fulbrook P. Core body temperature measurement: a comparison of axilla, tympanic membrane and pulmonary artery blood temperature. Intensive Crit Care Nurs 1997; 13: 266-72.

35 Myny D, De Waele J, Defloor T, et al. Temporal scanner thermometry: a new method of core temperature estimation in ICU patients. Scot Med J 2005; 50: 15-8.

36 Dahyot-Fizelier C, Lamarche S, Kerforne T, et al. Accuracy of zero-heat-flux cutaneous temperature in intensive care adults. Crit Care Med 2017; 45: e715-7.

37 Eshraghi Y, Nasr V, Parra-Sanchez I. An evaluation of a zeroheat-flux cutaneous thermometer in cardiac surgical patients. Anesth Analg 2014; 119: 543-9. 